\title{
Research on Time-Frequency Characteristics of Infrasound Information before rock Failure under Compression
}

\author{
Hong $\mathrm{Xu}^{1,2,3, \mathrm{a}^{*}}$, Tingqiang Zhou ${ }^{4}$ \\ ${ }^{1}$ Chongqing Key Laboratory of Exogenic Mineralization and Mine Environment, Chongqing \\ Institute of Geology and Mineral Resources, Chongqing 400042, China; \\ ${ }^{2}$ Chongqing Research Center of State Key Laboratory of Coal Resources and Safe Mining, \\ Chongqing, 400042, China; \\ ${ }^{3}$ Key Laboratory of New Technology for Construction of Cities in Mountain Area, \\ Chongqing University, 400045, Chongqing,China; \\ ${ }^{4}$ Chongqing three gorges university, wanzhou, 404000,China; \\ ${ }^{a}$ Email: njzy_fengyu@qq.com
}

Keywords: List the keywords covered in your paper. These keywords will also be used by the publisher to produce a keyword index.

Abstract. Six group of axial compression tests of sandstone were conducted in laboratory, and the infrasound signals of each test process were recorded synchronously, then the time-frequency characteristics of all signals have been analyzed to find the failure precursor information, the results show that: The infrasound of rock failure always manifests as two forms, one is persistent low amplitude signal and the other is spasmodic high amplitude signal, the two kind of infrasound emission forms may corresponding to the two process of micro cracks development in rock specimens, that is the random nucleartion process of cracks and the concentration and pooling nucleartion process of cracks, from the begin of loading to rock failure ,there always have two or three times of centralized high amplitude signal emission; also, the time-frequency analysis find that, there have different frequency components between low amplitude and high amplitude, the low amplitude signal has relatively single frequency components, the range of frequency mainly concentrated in $6 \sim 8 \mathrm{~Hz}$, the central frequency is about $7.5 \mathrm{~Hz}$, otherwise, the high amplitude signal has more complicated frequency components, it has three frequency parts, the lower frequency part concentrated in $2 \sim 6 \mathrm{~Hz}$, the central frequency is about $4.5 \mathrm{~Hz}$, the middle frequency part lies in $6 \sim 8 \mathrm{~Hz}$, the central frequency is about $7.5 \mathrm{~Hz}$, and the higher frequency part is ranged in $8 \sim 12 \mathrm{~Hz}$, the central frequency is about $9 \mathrm{~Hz}$ to $10 \mathrm{~Hz}$, different high amplitude signal always contains two or three frequency ranges above. The characteristics of infrasound waveform and its time-frequency above can be looked as an important mark of precursor infrasound emission by rock before failure.

\section{Introduction}

The infrasound is a special case of acoustic emission of rock during its deformation and failure. Since the Kaiser effect in rock was found by Goodman in the 1960's, many researches have been done by domestic and forging scholars and the results are very $\operatorname{rich}^{1 \sim 8]}$. There also have many achievements been successfully applied ${ }^{[9,10,11]}$. But from the exiting documents, we found that the previous studies on rock acoustic emission are almost focused on the high frequency signal represented by Kaiser effect, this kind of high frequency acoustic wave decays quickly in the air, and it can't achieve the remote monitoring effect .By contrast, the infrasound have obvious advantages on propagation distance and the ability to overcome obstacles.it provides a possible means for remote monitoring of rock failure.

There have little results and documents about infrasound characteristics of rock besides the following. In 1982, a destroy experiment with 35species of different rocks such as Granit and Marble was conducted in institute of earthquake research in Beingjing university of technology, and the 
infrasound was received intermittently during the rock stress process ${ }^{[12]}$. In the former Soviet Union, the researchers once did an uniaxial compression test of concrete with granite gravel on a fifty thousand tons of testing machine, the scale of test specimen is $0.55 \times 0.55 \times 0.65 \mathrm{~m}^{3}$, the infrasound wave had been detected during the compression course. In recent years, some new research work in this field has been carried out in Chengdu university of technology, the new achievement is as following: Made it clear that the phenomenon of highlight infrasound emission exists in the course of rock failure, and found the distribution range of excellent frequency of this kind of infrasound ${ }^{[13]}$. From the literature retrieval above, we can see that although there have some research results about infrasound of rock failure, but the research is not deep enough, especially, there's no research on the time and frequency characteristics of this infrasound. In fact, because the infrasound takes the air as transmission medium and the air is filled with much interference information, so the identification of rock infrasound is the primary aspect of related research and application, while to master the time and frequency characteristic of infrasound during rock failure is the basic of signal identification. Given this, we try to do some study on time and frequency characteristic of infrasound during rock failure in this paper, so as to make some reference and promoting on the further research and application in this field.

\section{Experimental process and analysis of the environmental noise}

In this experiment, the experimental samples are sandstone which is widely distributed in Chongqing area. Specimens were made into cylinder in accordance with the standard compressive test; there are 6 groups of specimens with diameter $50 \mathrm{~mm}$, height $100 \mathrm{~mm}$ are made for experiment. The test process was completed on the electro-hydraulic servo rock triaxial test machine in Chongqing Three Gorges University. The testing machine can carry out rock uniaxial and triaxial experiments, it can synchronize to collect rock time-load data and time-deformation data during the experiment. The CASI-ISM-2009 infrasound sensor and its matching digital recorder which produced by Chinese Academy of Science Institute of acoustics were selected to collect the infrasound information. Its working frequency range is $0.001 \sim 100 \mathrm{~Hz}$, and the measurement accuracy is $368 \mathrm{mV} / \mathrm{Pa}$. In the process of experiment, the infrasound sensors and recorders were connected together; the electrical signal from infrasound sensor was converted into digital signal by the digital recorder, and then transmitted to computer control and analysis system. The experiment was carried out in accordance with the conventional uniaxial compression experiment, no probe equipment is installed on the specimen surface, the infrasound sensor is about $1.5 \mathrm{~m}$ away from loading system. The infrasound data collection started at loading beginning and stopped at loading finish time. Infrasound data acquisition frequency is $1024 \mathrm{~Hz}$. Loading and infrasound collection system is shown in figure 1.
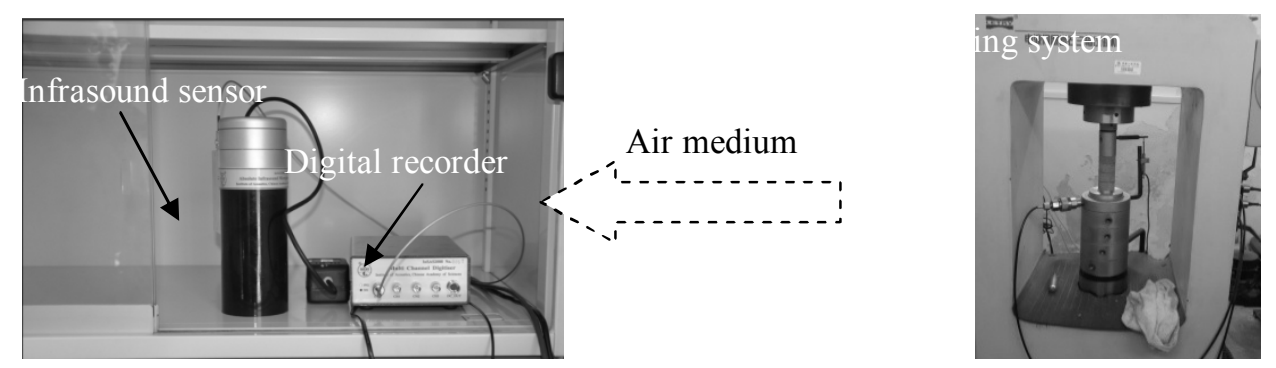

Fig.1 Load and infrasound collection system

Infrasound data in this experiment is gathered by non-contact way, so the air is the mainly transmission medium, and there are a lot of noise interference in the air. In order to obtain accurate rock damage infrasound information, it is necessary to analysis the laboratory noise. In this experiment, the ambient noise data was obtained with the following steps. First, open all of the experiment equipment without loading, second, use infrasound acquisition equipment to collect data after the device operation are stable, the acquisition time of ambient noise data is 20s.Figure 2 is the frequency spectrum of ambient noise. 


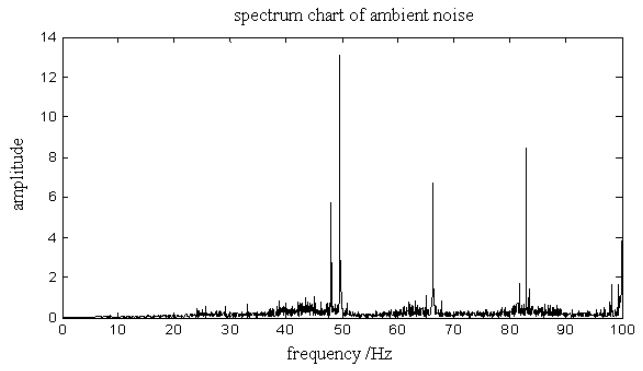

Fig.2 frequency distribution of ambient noise

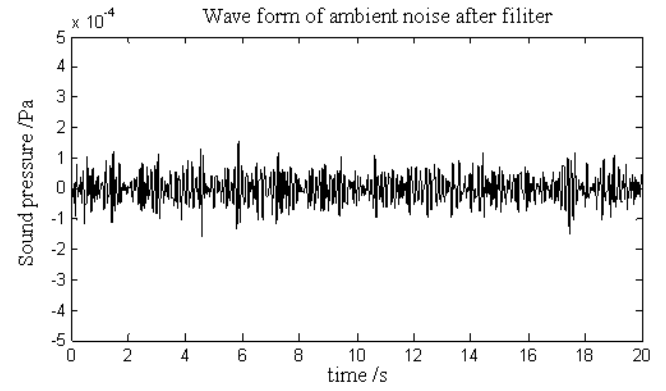

Fig.3 Waveform of ambient noise after filter

As can be seen from fig.2, the environment noise frequency is mainly concentrated in $50 \mathrm{~Hz}, 68 \mathrm{~Hz}$, $85 \mathrm{~Hz}$ and $100 \mathrm{~Hz}$, infrasound component of less than $20 \mathrm{~Hz}$ is pretty poor, Therefore, most of the ambient noise can be filter out if the low-pass filter with stop band width of $20 \mathrm{~Hz}$ is used, at the same time, in view of the research results in document [13], the frequency of infrasound during rock failure mainly focus below $10 \mathrm{~Hz}$. Based on the above two points, and combined with the actual needs of the experiment, the Butterworth low-pass filter was adopted to filter the environment noise, the pass band width is set to be $10 \mathrm{~Hz}$, stop band width is $20 \mathrm{~Hz}$, the maximum attenuation of pass band is $0.5 \mathrm{~dB}$, minimum attenuation of stop band is $40 \mathrm{~dB}$, waveform of ambient noise after filter is shown in figure 3.From figure 3 we can see that, the infrasound wave shape of environment is relatively stable, the sound pressure amplitude mostly concentrate between $+100 \mu \mathrm{Pa}$ and $-100 \mu \mathrm{Pa}$, no sudden change in amplitude, and maximum sound pressure amplitude is less than $150 \mu \mathrm{Pa}$.

\section{Analysis of time-varying characteristics of infrasound shape during rock failure}

A series of six experiments of sandstone specimens under compression was completed in this experiment, the constant rate with $1 \mathrm{KN} / \mathrm{s}$ was used in the process of loading. During the experiment process, the infrasound data and the displacement data are all collected, then the time-sound pressure graph and the time-displacement curve are drawn in a same chart, the result is as following:

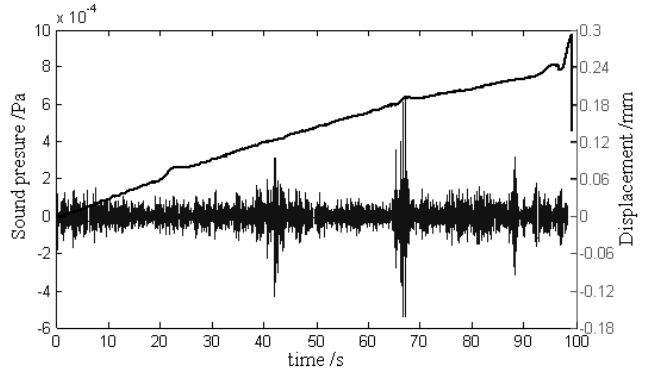

specimen $\mathrm{S} 1$

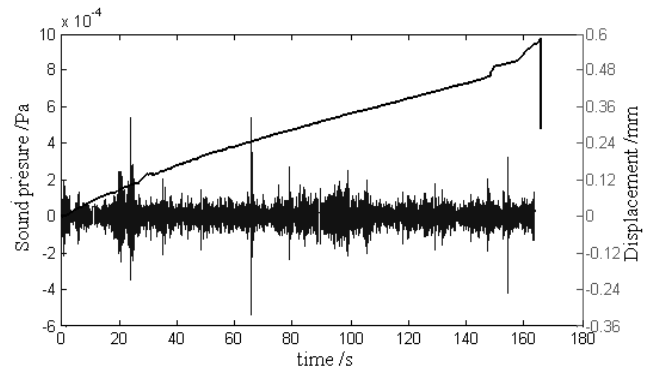

specimen S3

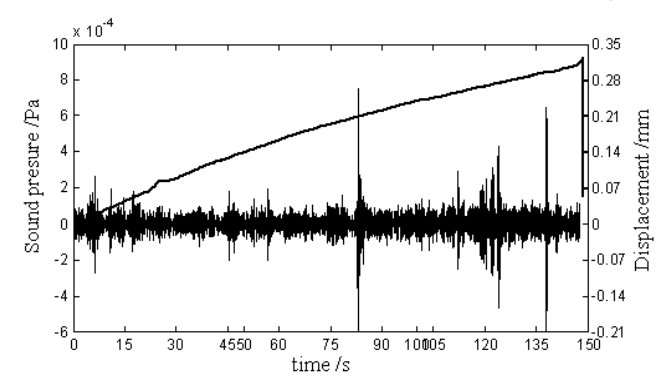

specimen $\mathrm{S} 2$

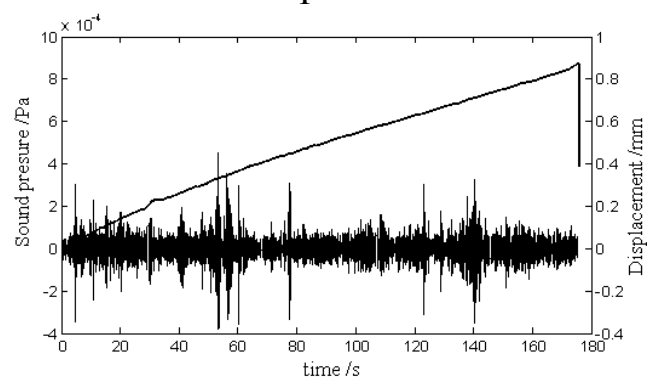

specimen S4 


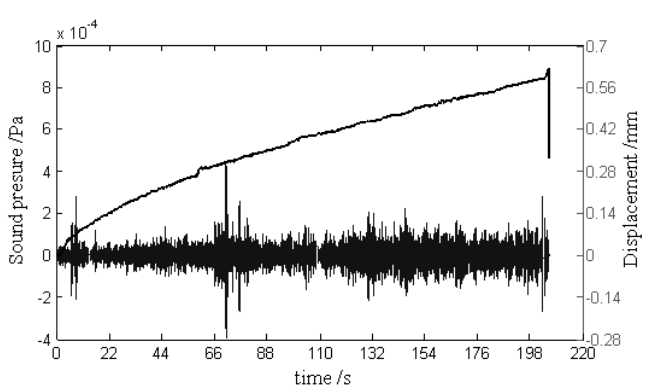

specimen S5

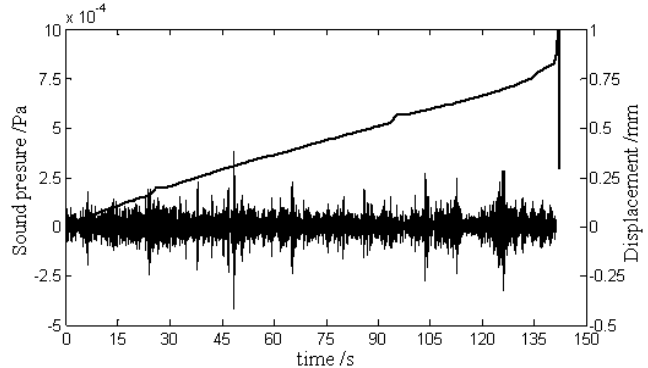

specimen $\mathrm{S} 6$

Fig.4 Waveform and Time-displacement curve of different specimens

From the above graph we can see that in the process of compressive deformation of rock specimens, the infrasound pulse signal did not show any significantly increasing or decreasing trend with the increasing of deformation, but perform a certain of periodicity, it is an obvious difference with the Kaiser effect of rock. There are some lies in the 6 specimens. First of all, from the view of wave form, we can find two basic emission forms of infrasound during deformation, one is the sustained low amplitude pulse signal and the other is the sudden high amplitude pulse. Secondly, from the perspective of time-varying process of infrasonic we find that the emission of infrasound consisted from the initial stage of elastic deformation to the end of deformation stage. The infrasound appears first at low sound pressure amplitude, and a concentration emission of high-strength infrasound will happen at regular intervals, it acts as the emergence of a small amount of high amplitude pulses, in the process of deformation start until destruction, there are usually three to four times concentrated emission of high amplitude infrasound, which reflects the dynamic process of damage development and agglomeration in rock specimens. Previous studies[14] shows the development of internal damage of rock, the damage begins first with the nucleation of random and isolated micro defects and then generate to micro cracks, with the distribution of micro defects nucleation nearly to saturate, micro defects turn into aggregating nucleation, permutation and growth then generate into large scale cracks. The infrasound pulse information obtained in this experiment perfectly reflects the above two processes, we have reason to believe that, in the waveform of infrasonic, persistent low amplitude signal may correspond to the random nucleation process of rock micro cracks, and high amplitude pulses reflect the process of aggregating nucleation and arrangement growth of micro defects. The formation of rock's final macroscopic damage surface is the result of multiply aggregation and growth of micro fracture.

\section{Time-varying characteristics of infrasound frequency}

In signal analysis field, Fourier transformation is an effective tool for signal conversion from the time domain to the frequency domain, but it cannot reflect the behavior of signal frequency changing with the time, so it only fits to stable signal. In order to deal with the contradiction of localization of frequency domain and time domain, time domain localization methods have been proposed, the most typical method is the short-time Fourier analysis which proposed by Gabor in 1946, the basic idea is to multiply a time limited window function before fourier transform, and assume that the non-stationary signal analysis in time window interval is stationary, when the window slip on time axis the signal is put into analysis state piecewise, thus the time-varying characteristic of signal can be obtained ${ }^{[15]}$. For rock damage, at different times and stress, the pattern, degree and number of its internal damage are not the same, and the acoustic emission method and frequency will change accordingly, so the signal is non-stationary, the short-time Fourier transform can reflect this characteristic more vivid. Given this, the short-time Fourier transformation method was adopted in this paper, the Specgram function in Matlab was used to analysis time-frequency characteristics of the infrasound during the failure process of rock, and the hamming window was chosen in analysis, the window length is set to be 1024 which is 
consistent with sampling length, diagram of time-frequency characteristics of infrasound by different specimens is shown in figure 5 .

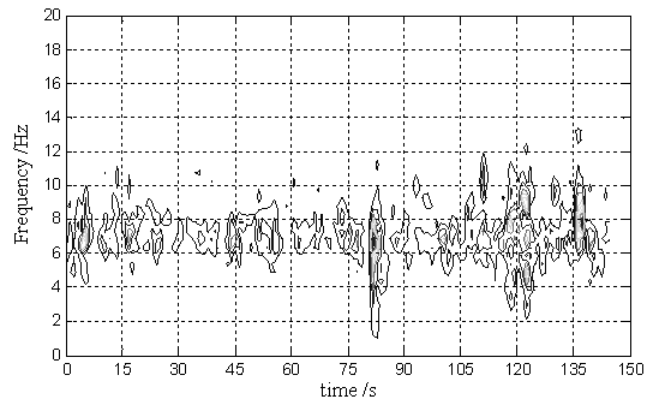

Specimen S1

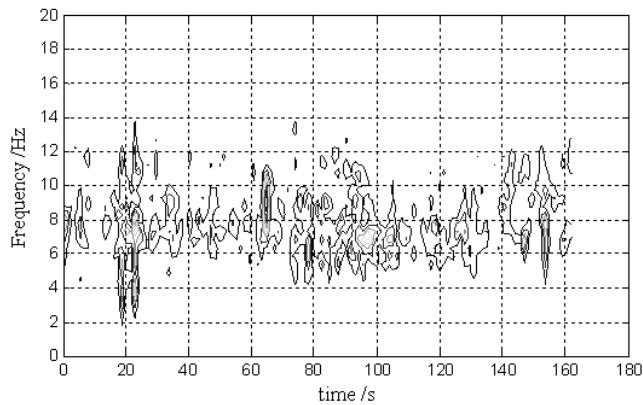

specimen S3

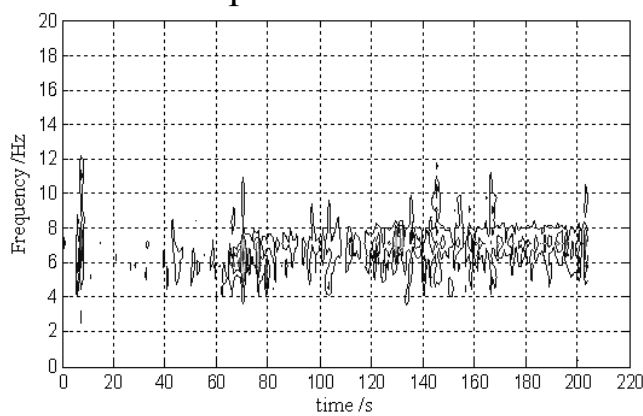

specimen S5

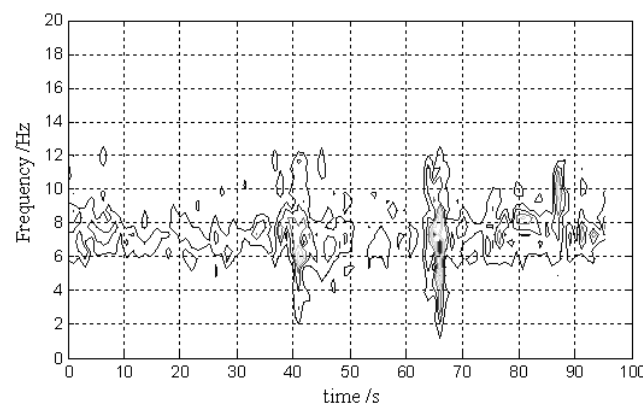

Specimen S2

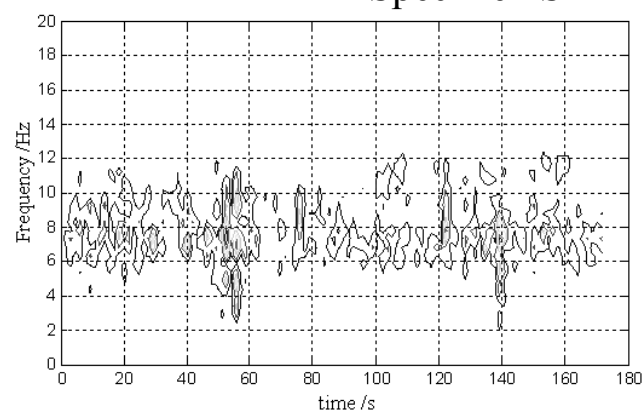

specimen S4

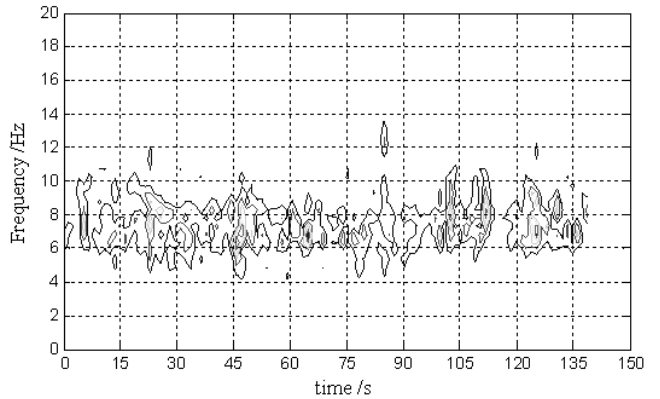

specimen S6

Fig.5 Time-frequency characteristics of infrasound by different specimens

The time-frequency characteristics of 6 specimens reflect a common law, namely, the frequency of low amplitude infrasound signal has relative single component and narrow frequency range, and high amplitude impulses have more complex frequency components, it usually has two to three frequency center, the frequency distribution range is also wider. Contrasting the time-frequency diagram and the waveform diagram we found that the signal amplitude and frequency distribution has obvious corresponding relationship in time. Take specimen S1 for example, in the following time periods such as $0 \mathrm{~s} \sim 80 \mathrm{~s} 、 90 \mathrm{~s} \sim 110 \mathrm{~s}, 125 \mathrm{~s} \sim 135 \mathrm{~s}$, infrasound signal mainly characterized by continuous low amplitude pulse, it can be clearly seen from the time-frequency graph that signal frequency components is relatively single in these periods, frequency range is mainly concentrated between $6 \sim 8 \mathrm{~Hz}$, the center frequency is about $7.0 \mathrm{~Hz}$. In the whole process of loading, specimen has gone through three times of concentrated emission process of high amplitude infrasound, respectively near in $80 \mathrm{~s}, 120 \mathrm{~s}$ and $140 \mathrm{~s}$; these three relatively concentrated high amplitude impulses has obvious different frequency components with the low amplitude impulse before. It contains wider frequency range and more complex frequency components. Time-frequency maps shows, for the first high amplitude signal that appeared near $80 \mathrm{~s}$, its frequency is mainly concentrated in $2 \sim 10 \mathrm{~Hz}$, the center frequency is about $6.5 \mathrm{~Hz}$, and has high strength signal duration within $4 \sim 8 \mathrm{~Hz}$; for the high amplitude signal that appeared near $120 \mathrm{~s}$, its frequency range is from $4 \mathrm{~Hz}$ to $12 \mathrm{~Hz}$, the signal is divided into four different frequency ranges, the lower frequency ranges take $4.5 \mathrm{~Hz}$ as distribution center, focused on $4 \sim 6 \mathrm{~Hz}$; The 
intermediate frequencies take $7 \mathrm{~Hz}$ as center, mainly focused on $6.5 \sim 7.5 \mathrm{~Hz}$, and the higher frequencies is located between $8 \mathrm{~Hz}$ and $10 \mathrm{~Hz}$, the center frequency is about $9 \mathrm{~Hz}$. In addition, for this high amplitude signals emission, there still has a signal with frequency center $10.5 \mathrm{~Hz}$, and frequency range $9 \sim 11 \mathrm{~Hz}$. In the third time of high amplitude pulse emission, the frequency range is $6 \sim 10 \mathrm{~Hz}$, center frequency is $8 \mathrm{~Hz}$.

Table 1 is the distribution of frequency characteristic of 6 specimens, it can be seen from table 1 , time-frequency analysis results of 6 specimens have the same regularity.

Tab.1 Frequency distribution of differen specimens $\quad$ Unit: $\mathrm{Hz}$

\begin{tabular}{|c|c|c|c|c|c|c|c|c|c|c|}
\hline \multirow{2}{*}{$\begin{array}{c}\text { Specimen } \\
\text { No }\end{array}$} & \multicolumn{2}{|c|}{ Low amplitude } & \multicolumn{7}{|c|}{ High amplitude } \\
\cline { 2 - 12 } & range & center & \multicolumn{2}{|c|}{ First emission } & \multicolumn{2}{|c|}{ Second emission } & \multicolumn{2}{|c|}{ Third mission } & \multicolumn{2}{c|}{ Fourth emission } \\
\cline { 4 - 12 } & range & center & range & center & range & center & range & center \\
\hline S1 & $6 \sim 8$ & 7.0 & $2 \sim 10$ & 6.5 & $4 \sim 12$ & $4.5,7.0,9.0,10.5$ & $6 \sim 10$ & 8.0 & & \\
\hline S2 & $5 \sim 8$ & 7.5 & $4 \sim 8$ & 6.0 & $2 \sim 9$ & $4.5,7.0$ & $8 \sim 11$ & 10.0 & & \\
\hline S3 & $6 \sim 8$ & 7.5 & $3 \sim 11$ & $4.5,7.5,9.5$ & $8 \sim 10$ & 8.5 & $7 \sim 10$ & 8.0 & $4 \sim 9$ & $5.0,7.5$ \\
\hline S4 & $6 \sim 8$ & 7.0 & $2 \sim 12$ & $4.0,8.0,11,0$ & $7 \sim 11$ & 9.0 & $5 \sim 9$ & $6.5,8.0$ & & \\
\hline S5 & $6 \sim 8$ & 6.5 & $5 \sim 9$ & 6.5 & $7 \sim 10$ & 8.0 & $7 \sim 11$ & $7.0,9.0$ & & \\
\hline S6 & $6 \sim 8$ & 7.0 & $4 \sim 12$ & $4.5,7.0,11.0$ & $5 \sim 11$ & $5.0,6.5$ & & & & \\
\hline
\end{tabular}

The frequency difference in different stages of infrasonic reflects the force mechanism of rock internal damages in the process of initiation, propagation and aggregating nucleation. As mentioned before, low amplitude pulse signals corresponding to the random nucleation process of specimen's internal micro cracks, previous studies indicated that, in this process, the shear slip and dislocation between mineral grains play a leading role, the stress on crack tip is mainly tensile stress and the stress form is relatively single, thus it plays a relatively single and stable frequency range in the time-frequency diagram of infrasonic. Different from it, the process of cracks entralized arrangement and aggregating nucleation is more complex, this process has not only tensile failure, but also include destructive action of shear and friction, and it shows more wide frequency range and more frequency center in the time-frequency graph .

\section{Conclusions}

1. Rock in the process of compressive deformation, can produce infrasound, infrasound emission is mainly in two basic forms, one is the persistent low amplitude pulse and the other is sudden high amplitude pulse, these two forms correspond to the different development stages of cracks respectively. The development and evolution law of the internal microscopic cracks in rock during damage can be more clearly reflected through the analysis of infrasound. It has a broad prospect in rock disaster early warming by use of infrasound monitoring method.

2.There usually have two to three times of high amplitude pulse concentrated emission, during the process of rock forcing till damage. The maximum amplitude value of infrasound has little corresponding relationship with rock strength, but has a strong relationship with the anti-deformation ability of specimens, the stronger anti-deformation ability the specimen has, the higher maximum amplitude value the infrasound signal contains.

3.Center frequency of sustained low amplitude pulse mainly concentrated in $6 \sim 8 \mathrm{~Hz}$, the frequency range is relatively stable, high amplitude pulse has wider frequency range and more complex frequency components, its frequency is in the range of $2 \sim 12 \mathrm{~Hz}$, usually with three frequency bands of high, 
medium and low, the high frequency band is $8 \sim 12 \mathrm{~Hz}$, the median is $6 \sim 8 \mathrm{~Hz}$, and the low is $2 \sim 6 \mathrm{~Hz}$. According to different stress mechanism, arbitrarily high amplitude signals usually contain two to three frequency bands above.

4. These time-frequency Characteristics of infrasound during rock deformation provide an important basis for the identification of infrasound before rock failure.

\section{Acknowledgements}

This work is supported by the National Nature Science Foundation of China (Grant No : 41202252)

\section{Reference}

[1] Goodman R.E, Geol.Soc.Am.Bull.1963 (74)487 490.

[2] Fleischmann,P,Rouby,D.Lakestani,f.and Baboux,j.C. A Spectrum analysis of acoustic mission[J]. Non-Destructive Testing,8(1975),No.5,241 244.

[3] Boyce G M.A study of the acoustic emission response of warious rock type [D].Drexel University 1981.

[4] SHIOTANIT,OHTSU M,IKEDA K. Detection and evaluation of AE Waves due to rock[J]. Construction and Building Materials,2001,15(5):235 246.

[5] Li Shulin,Yin Xiangang,Wang Yongjia,etc. Studies of Acoustic Emission Characteristics of Uniaxial compressive rock failure[J].Chinese Journal of Rock Mechanics and Engineering,2004, 23(15):2499 2503. (in Chinese)

[6] Xu Dongqiang,San Xiaoyun, Zen Zaixue. Analysis of damage mechanics on Acoustic Emission Characteristics of Rock under Biaxial Compression[J].Ground Pressure and Strata Control, 2000,N03:82 84. (in Chinese)

[7] Xiang Tianbing,Feng Xiating,Chen Bingrui,etc.Rock Failure Mechanism and Tre Triaxial Experimental Study of Specimens with Single Structural Plane under Three-dimensional Stress[J].Rock and Soil Mechanics, 2009,30(10):2908 2916.(in Chinese)

[8] Gong Yuxin, He Manchao,Wang Zhenghong,etc.Research on Time-Frequency Analysis Gorithm and Instantaneous Frequency Precursors for Acoustic Emission Data From Rock Failure Experimet[J].Chinese Journal of Rock Mechanics and Engineering, 2013,32(4):787 799.(in Chinese)

[9] YING Xiangang. A case of prediction of instable rock by acoustic emission[J].Journal of the China Railway Society ,2005,27(1):84 89.(in Chinese).

[10] ZHANG Guangqing,JIN Yan,CHEN Mian, Measurement of in-situ stresses by Kaiser Effect under confining pressures[J].Chinese Journal of Rock Mechanics and Engineering,2002,21(3): 360 363. (in Chinese)

[11] He Chunlin,Cui Dongliang. Application of multichannel acoustic emission system in the monitoring of mined-out area stability[J].Nonferrous Metals,2008,60(1):34 37.(in Chinese)

12] Li Junzhi,Cao Ming, Xia Yaqing.et al. An Experimental Study on Rock Compressing and Emission of Electromagnetic Waves before Earghquake[J].Journal of Beijing University of Technology, 1982,No4.47 53.

[13] Zhu Xing, Xu Qiang,Tang Minggao,etc.Experimental Study of Infrasound Wave Generated by Typical rock Fracture[J].Rock and Soil Mechanics, ,2013,34(5):1306 1311.(in Chinese) 
[14] Wang Li, Mao Yuanchun, Ye Jinsheng,etc. Models for Microcracks extension and damage Evolution Based on Number Series of Microdefects Nucleation[J]. Engineering Mechanics, 2013,30(8):278 286.(in Chinese)

[15] Ge Zhexue,Chen Zhongsheng. Technology and Application of Time-Frequency analysis by Use of Matlab[M].Beijing, Post and Telecom Press,2006.1.(in Chinese) 